\title{
Spectroscopic and photometric observations of the short-period RS CVn-type star ER Vulpeculae ${ }^{\star}$
}

\author{
D. P. Kjurkchieva ${ }^{1}$, D. V. Marchev ${ }^{1}$, and S. Zola ${ }^{2,3}$ \\ ${ }^{1}$ Department of Physics, Shoumen University, 9700 Shoumen, Bulgaria \\ and Isaac Newton Institute of Chile, Bulgarian Branch \\ e-mail: d.kyurkchieva@shu-bg.net; d.marchev@shu-bg.net \\ 2 Astronomical Observatory, Jagiellonian University, ul. Orla 171, 30-244 Cracow, Poland \\ 3 Mt. Suhora Observatory of the Pedagogical University, ul. Podchorazych 2, 30-084 Cracow, Poland
}

Received 10 September 2002 / Accepted 25 March 2003

\begin{abstract}
Spectroscopic observations around the $\mathrm{H}_{\alpha}$ line and BVR photometry of the eclipsing, short-period, RS CVn-type star ER Vul are presented. The solutions of new radial velocity and light curves yielded the following masses and radii of the components: $M_{1}=1.16 M_{\odot}, M_{2}=1.05 M_{\odot}, R_{1}=1.25 R_{\odot}, R_{2}=1.12 R_{\odot}$. The equatorial velocities corresponding to the measured rotational broadenings of the spectral lines are: $V_{\mathrm{eq}}^{1}=90 \mathrm{~km} \mathrm{~s}^{-1}$ and $V_{\mathrm{eq}}^{2}=80 \mathrm{~km} \mathrm{~s}^{-1}$. Their ratio is just equal to the ratio of the stellar radii. We detected excess emission in the $\mathrm{H}_{\alpha}$ and CaI 6494 lines that changes during the orbital cycle. Our spectral data show the presence of absorbing matter around the mass center of the system.
\end{abstract}

Key words. stars: activity - binaries: eclipsing - binaries: spectroscopic - stars: chromospheres - stars: individual: ER Vul stars: starspots

\section{Introduction}

The close, eclipsing binary system ER Vul offers special opportunities to investigate the activity in sun-like stars. Although the components of ER Vul are very similar to the Sun in global parameters, their rotation is faster by a factor of 40 and, correspondingly, a high level of activity may be expected. It can be also an appropriate target to check the validity of an extrapolation of the solar paradigm to the rapidly rotating active stars.

Different indicators of stellar activity are introduced by analogy to the Sun. The great number of the stars allows to search for relationships between the spatial, time and energetic scales of their activity appearances and global parameters (for instance the Wilson-Bappu effect). On the other hand, the study of the stellar activity is a basis for improvement of the magnetic-dynamo theory and for establishment of the criteria for solar forecast. Enhanced emission cores in the $\mathrm{CaII} \mathrm{H}$ and $\mathrm{K}$ lines are the primary optical indicators of chromospheric activity. The activity levels can be also determined by the presence of pure emission or filled-in cores of the $\mathrm{H}_{\alpha}$ line, $\mathrm{MgI}$ lines, etc. because there are good correlations among the different activity indicators.

Send offprint requests to: S. Zola, e-mail: zola@astro1.as.wsp.krakow.pl

* Based on spectral observations collected at the National Astronomical Observatory, Bulgaria.
On the basis of the emission cores of the CaII H and $\mathrm{K}$ lines and a variable light curve, Hall (1976) classified ER Vul as a member of the short-period group of RS CVn systems. The variabilities of ER Vul, at different spectral ranges, have been extensively reviewed (e.g., Olah et al. 1994) and need not be repeated here. The light variations were attributed to the presence of spots, however, some investigators (McLean 1982; Hill et al. 1990; Arevalo et al. 1988; Zeinali et al. 1995) argued that the existence of circumstellar material (cloud at the inner Lagrangian point or a gas stream between the components) is a more likely explanation for the observed photometric and spectroscopic peculiarities of ER Vul.

Lazaro \& Arevalo (1997) found excess emission from both components of ER Vul in the Balmer $\mathrm{H}_{\alpha}$ and $\mathrm{H}_{\beta}$ lines as well as in the CaII IRT lines (8498 and $8542 \AA$ ), which total emission fluxes change during the orbital cycle almost as the brightness of the system does. Lazaro \& Arevalo (1997) attributed both the slightly stronger emission excesses around the second quadrature and the weaker emission excesses at the secondary eclipse to a larger emission from the secondary star. Excess emission in the lines $\mathrm{H}_{\alpha}$, CaII IRT and MgIb $(5167.3,5172.7,5183.6 \AA)$ was detected also by Gunn \& Doyle (1997) with indications that the secondary star is the more active component.

Vilhu \& Rucinski (1983) measured fluxes of various chromospheric and transition region lines in the IUE spectra of ER Vul and concluded that they were on the borderline between those expected for detached and contact systems. The EUVE 


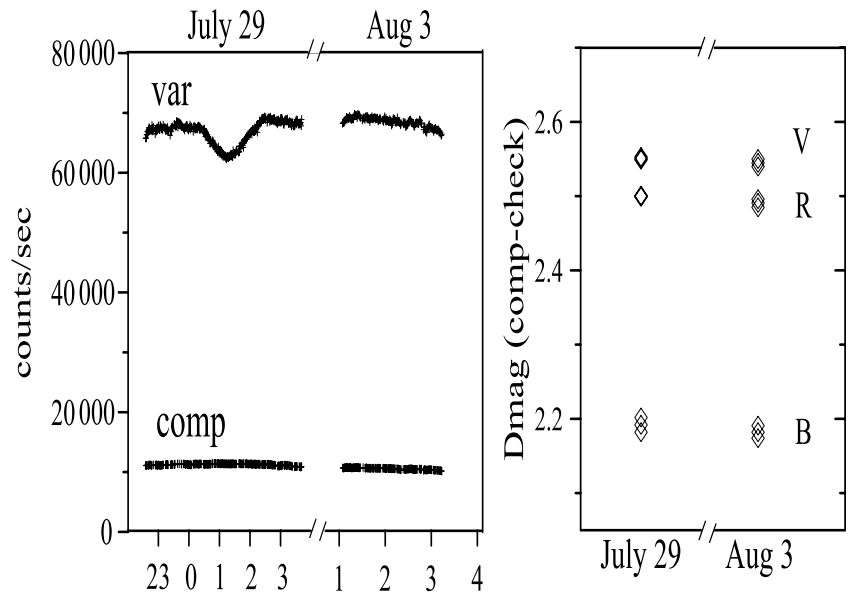

Fig. 1. Illustration of the constancy of the comparison star.

observations also revealed emission lines typical of chromospheric, transition and low corona regions (Rucinski 1998).

The aim of our photometric and spectral observations of ER Vul is to search for appearances of its activity and to re-determine its global parameters.

\section{Observations}

During 6 nights at the end of July and beginning of August 1999, the $B V R$ photometry of ER Vul was obtained with a twochannel photometer mounted on the $60-\mathrm{cm}$ telescope (Kreiner et al. 1993) at the Mt. Suhora Observatory. The telescope is equipped with an autoguider (Krzesinski \& Wojcik 1993). The cross-calibration of the channels was made every night. Due to the limited area of the two-channel photometer we used

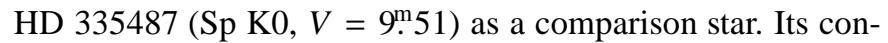
stancy was checked against the star HD 199375 (Sp K2). In Fig. 1 we present the behavior of our comparison star. The left panel shows raw measurements and no short scale variations can be seen, just the effect of atmospheric extinction. The right panel shows differential magnitudes between the comparison and check stars. We found the comparison star constant to within 0.01 mag during our observations. The integration times were $10 \mathrm{~s}$ in all colors. The errors of the individual points do not exceed 0 . 01 in $V$ and $R_{\mathrm{C}}$ filters and 0.015 in the $B$ filter.

The data were phased according to the ephemeris (Kreiner et al. 2001)

$$
H J D(M i n I)=2440182.2593+0.69809479 * E
$$

and left in the instrumental system. Figure 2 presents the complete light curve, where observations done during different nights are marked by different symbols.

ER Vul was observed also spectroscopically (resolution $0.19 \AA /$ pixel) during two consecutive nights in August 2001 around the $\mathrm{H}_{\alpha}$ line, which is a spectroscopic indicator of stellar chromospheric activity (Zarro \& Rogers 1983; Frasca \& Catalano 1994; Strassmeier et al. 1990). We used the Photometrics AT200 CCD Camera with SITe SI003AB $1024 \times 1024$ pixel chip mounted on the Coude spectrograph (grating $B \& L 632 / 14.7^{\circ}$ ) of the $2-\mathrm{m}$ telescope of the National

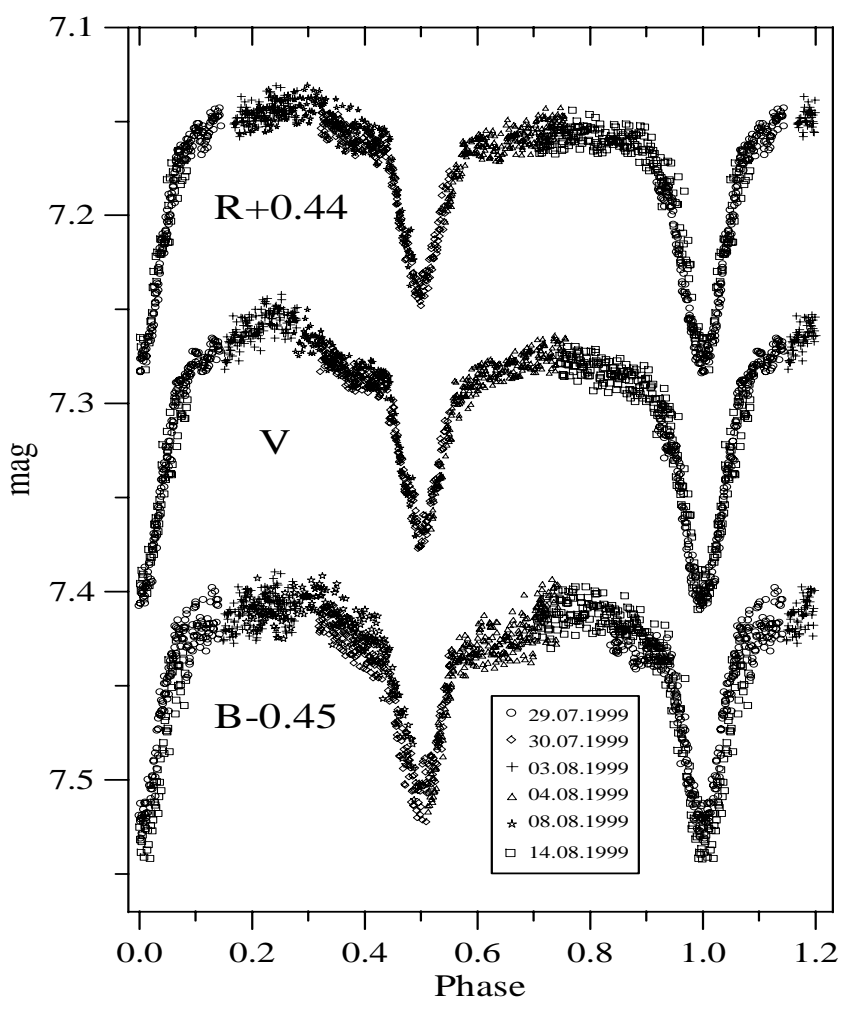

Fig. 2. The light curve of ER Vul in the instrumental system. Observations gathered in different nights are denoted by different symbols.

Astronomical Observatory at Rozhen. The seeing during the observations did not exceed $2 \operatorname{arcsec}(F W H M)$. The exposure time was $20 \mathrm{~min}$ and the $S / N$ ratio was in the range 100-180. The bias frames and flat-field integrations were obtained at the beginning and end of each night. All star's integrations were alternated with the Th-Ar comparison source exposures. The data were processed in a standard way (bias substraction, flatfield division and wave-length calibration) using the PCIPS (Smirnov et al. 1992) and Rewia (Borkowski 1988) software packages.

In the observed range $6465-6665 \AA$, besides of the $\mathrm{H}_{\alpha}$ line, there are several weaker lines showing orbital variability (Fig. 3). The profiles of the $\mathrm{H}_{\alpha}$ are shown in Fig. 4, while FeI 6593 and CaI 6494 lines are displayed in Figs. 8 and 9 together with the corresponding orbital phases calculated according to the ephemeris (1). An appropriate Fourier noise filter was applied in order to remove the high-frequency noise. This procedure causes some smoothing of the profiles without loss of their important features (Gray 1992).

\section{Analysis of the spectral data}

\subsection{Orbital variability of the spectral lines}

In order to get information about the spatial location of the active regions in ER Vul we analyzed the behavior of the spectral lines at different orbital phases.

The observed $\mathrm{H}_{\alpha}$ lines of the two stellar components are always in absorption. Third absorption feature T (Fig. 4) superposes the $\mathrm{H}_{\alpha}$ lines of both stars out of the eclipses. It causes 


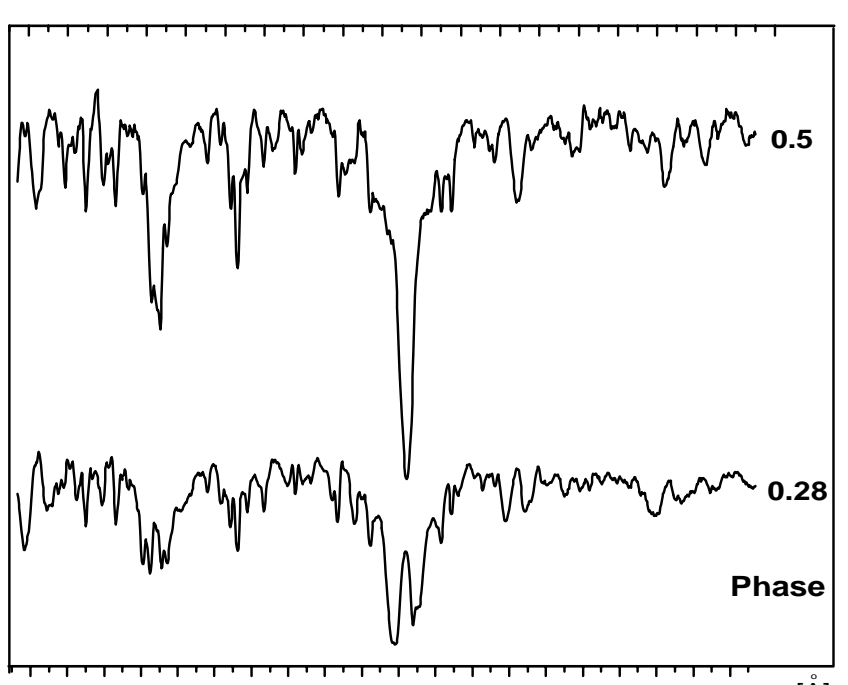

$6470649065106530655065706590 \quad 661066306650$

$[\AA]$

Fig. 3. The spectra of ER Vul at the secondary eclipse and at the first quadrature.

distortions of the profiles and probably could be a reason why the line of the secondary star seems deeper than that of the primary at the phase range $0.08-0.12$. Such a feature was not detected in the spectra of CG Cyg which we observed during the same nights (Kjurkchieva et al. 2003). Because this absorption feature almost does not shift during the orbital cycle and it is invisible just in the middle of the eclipses it could be attributed to some absorbing matter around the mass center of the binary system.

An emission feature E (Fig. 5) on the blue wing of the $\mathrm{H}_{\alpha}$ line appears during both eclipses (phases $0.41-0.57$ and 0.92-0.10). It may be well distinguished (see Fig. 3) by comparison of the $\mathrm{H} \alpha$ profiles at the secondary eclipse and the first quadrature. Similar (but weaker) feature is visible also in the spectra of Lazaro \& Arevalo (1997) during the secondary eclipse.

The $\mathrm{H}_{\alpha}$ profiles in the middle of the primary and secondary eclipses agree well both in shape and in depth (Fig. 5). The $\mathrm{H} \alpha$ profile observed by Lazaro \& Arevalo (1997) at the secondary eclipse is deeper (by around $10 \%$ ) than that at the primary one. The depth of the $\mathrm{H}_{\alpha}$ line we observed at both eclipses is almost equal to that detected by Lazaro \& Arevalo (1997) and Gunn \& Doyle (1997) at the primary eclipse. The equality of the $\mathrm{H}_{\alpha}$ profiles that we observed at both eclipses means that there were no difference between the $\mathrm{H}_{\alpha}$ absorption/emission properties of the stellar surfaces visible at these phases.

In contrast, the $\mathrm{H}_{\alpha}$ profiles around the quadratures differ (Fig. 6). The line of the primary star is deeper around the second quadrature than at the first one. This fact could be attributed to an enhanced $\mathrm{H}_{\alpha}$ emission excess from its hemisphere visible around the first quadrature. It may be considered as another confirmation of the common trend of the spectral lines in the RS CVn-type stars: they tend to be stronger around the second quadrature than around the first one ("second quadrature effect", Kjurkchieva et al. 2001).

The $\mathrm{H}_{\alpha}$ profiles of the secondary star seem almost the same around both quadratures (Fig. 6) but we should point out that the asymmetry at phase 0.22 is caused by the third absorption feature $\mathrm{T}$ while the distortion of the profile at phase 0.68 is due to the filled-in core (more apparent at phase 0.65 as seen in Fig. 4).

More information about the spatial distribution of the regions of the $\mathrm{H}_{\alpha}$ emission excess could be obtained from the variability of the equivalent width $(E W)$ of the line. The results of our measurements of the $E W$ of the total (absorption) $\mathrm{H}_{\alpha}$ profile (in $\AA$ units) at different phases are shown in Fig. 7. It is seen that the $E W$ 's are bigger at the eclipses (about $2.25 \AA$ ) than around the quadratures (near $1.8 \AA$ ). This phase variability of the total $E W$ of the $\mathrm{H}_{\alpha}$ absorption in ER Vul (Fig. 7) could be explained by bigger total $\mathrm{H}_{\alpha}$ emission excess out-of-eclipses than during the eclipses. The same phase trend of the total $\mathrm{H}_{\alpha}$ emission excess was established by Lazaro \& Arevalo (1997).

The mean value of the $E W$ of the total absorption during the orbital cycle is around $2 \AA$. It is considerably smaller than $E W$ of the $\mathrm{H}_{\alpha}$ absorption lines of the inactive stars $16 \mathrm{CncC}$ $(\mathrm{G} 5, E W=3.6 \AA)$ and $\beta \operatorname{Vir}(\mathrm{F} 9, E W=3.5 \AA)$, which we observed with the same equipment 8 months later, and the difference could be attributed to the $\mathrm{H}_{\alpha}$ emission excess of the stellar components in ER Vul (comparison of the observed spectrum of the active star with a spectrum of an inactive star is the basis of the spectral substraction technique, Gunn \& Hill 1997; Lazaro \& Arevalo 1977; Frasca et al. 2000).

In order to determine the contribution of each stellar component to the total $\mathrm{H}_{\alpha}$ emission excess one needs the $E W$ of their line profiles. The procedure of such a measurement is not well-defined mainly due to the wide wings of the $\mathrm{H}_{\alpha}$ line and their superposing in the case of binary system. That is why different $\mathrm{H}_{\alpha}$ activity indices (tightly correlated) have been used by different authors: $F W H M$, residual intensity $R_{\mathrm{c}}$, equivalent width of the excess emission $E W\left(\mathrm{H}_{\alpha}\right), \mathrm{H}_{\alpha}$ core flux $F(1.7 \AA)$ (Montes et al. 1995). Taking into account that the chromospheric emission is concentrated in the core of the $\mathrm{H}_{\alpha}$ line, we assumed that the measurement of the $E W$ of the stellar profiles below a certain level beneath the wide $\mathrm{H}_{\alpha}$ wings could provide adequate information about the relative contribution of the two stellar components to the total excess emission, as well as about their variability during the cycle. In this case, we measured the $E W$ of the $\mathrm{H}_{\alpha}$ lines of both components below the level 0.91 at phases 0.32 and 0.68 (symmetric to the secondary eclipse). The contribution of the third absorption $\mathrm{T}$ was removed in these $E W$ measurements. The obtained results $\left(E W_{1}(0.32)=0.27\right.$; $\left.E W_{2}(0.32)=0.16 ; E W_{1}(0.68)=0.28 ; E W_{2}(0.68)=0.10 \AA\right)$ mean that the main contributor to the $\mathrm{H}_{\alpha}$ emission excess at phase 0.68 is the secondary star.

The FeI 6593 line has no wide wings and the profiles originating from both stars are well separated out of the eclipses (Fig. 8). The shape of these profiles is similar to that of $\mathrm{H}_{\alpha}$ at the same phases. Third absorption feature T (Fig. 8) is visible at some phases out of eclipses. It is probably the reason why the profiles of the secondary seem deeper than those of the primary around the first quadrature (phase range 0.32-0.41).

The second (in strength) spectral feature with orbital variability in the observed spectral range is that around $6494 \AA$, displayed in Fig. 9. This feature has not been discussed in previous spectral investigations although its doubling is visible in the spectra of Lazaro \& Arevalo (1997). We suppose that this 

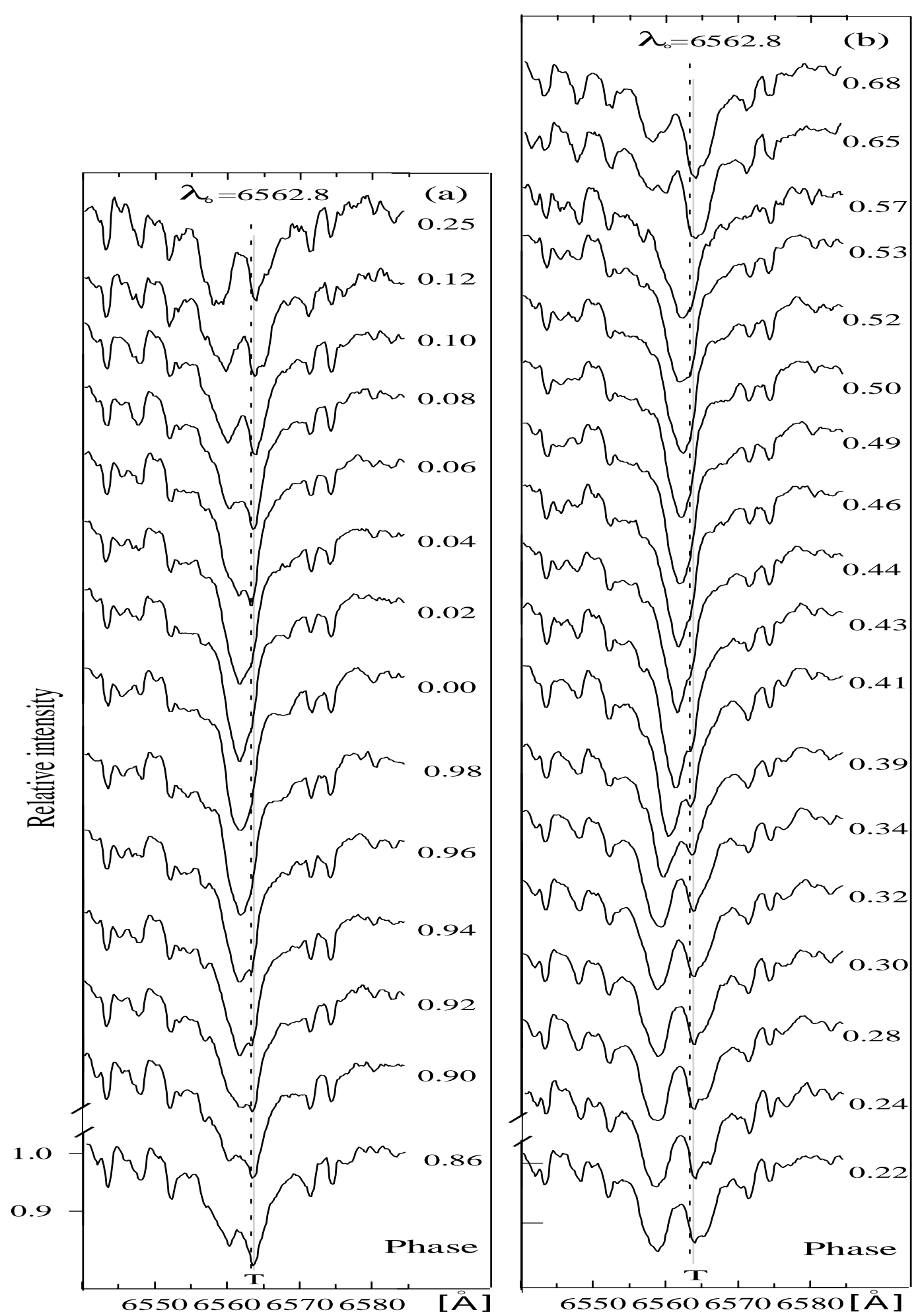

Fig. 4. $\mathrm{H}_{\alpha}$ profiles of ER Vul from August 14 (a - left panel) and August 15 (b-right panel). The position of the third absorption feature "T" is marked with a grey bar.

spectral feature represents the $\mathrm{CaI} 6493.7 \AA$ line blended by FeI $6494.5 \AA$ A. The $6494 \AA$ line has wide wings similarly to both the $\mathrm{H}_{\alpha}$ line (Fig. 3) and to most Ca lines (likely due to the chromospheric stratification). Figure 10 shows the wide profiles of the $\mathrm{H}_{\alpha}$ and CaI 6494 lines of ER Vul at the same phases. The similarity in the shape of both lines is apparent.
The cores of the CaI 6494 line are filled-in by emission at all phases (Fig. 9). It is difficult to get the precise quantitative estimation of this excess emission and to study its orbital variability due to the presence of telluric features. Figure 11 shows the CaI 6494 line in the spectra of the short-period, RS CVn-stars: CG Cyg (Kjurkchieva et al. 2003), ER Vul (this 


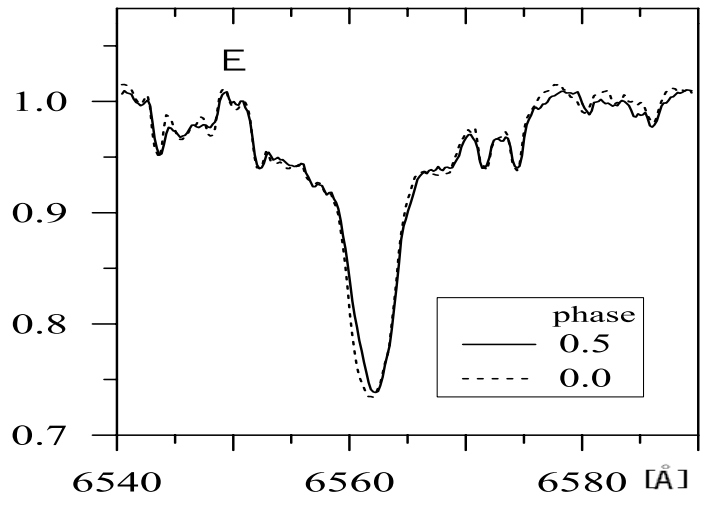

Fig. 5. $\mathrm{H}_{\alpha}$ line of ER Vul at primary (dashed line) and the secondary (continuous line) eclipses. "E" marks the emission feature on the blue wing of the line.

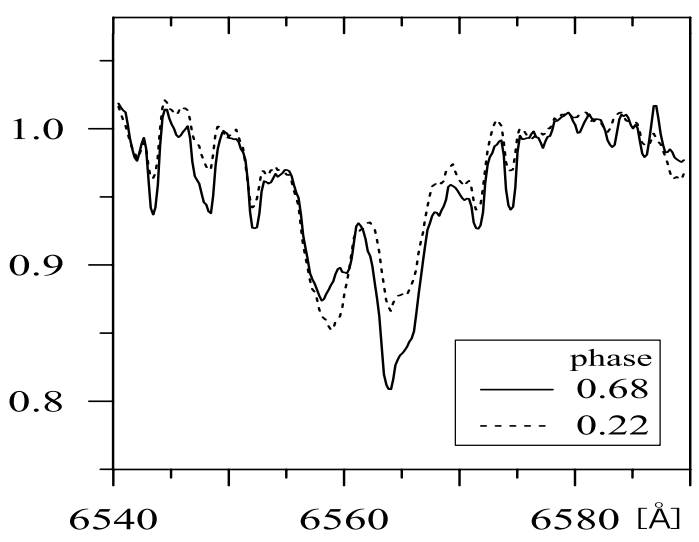

Fig. 6. The $\mathrm{H}_{\alpha}$ profiles of ER Vul around the quadratures.

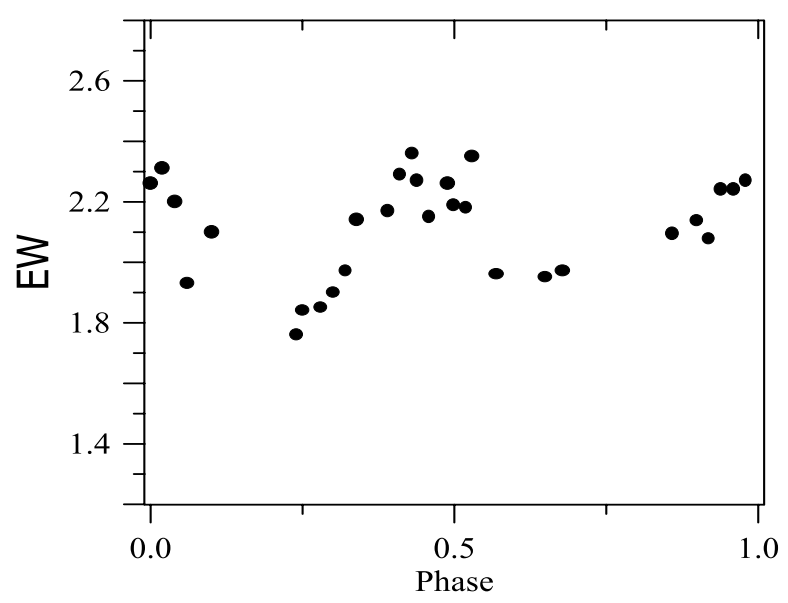

Fig. 7. $E W$ variation of the total $\mathrm{H}_{\alpha}$ absorption in the orbital phase.

paper) and WY Cnc (in preparation) at the same phases: just in the middle of the primary eclipse (phase 0 ) and slightly before the secondary minimum (phase 0.43 ). Emission excess visible in the central parts of all profiles appears as filled-in or emission core that is more apparent at the secondary eclipses. We noted that the CaI 6494 lines of all three stars are deeper at the primary eclipse than at the secondary one. If this fact is not accidental it could mean the higher emission excess in the CaI 6494 line from the hotter primaries in these binary systems
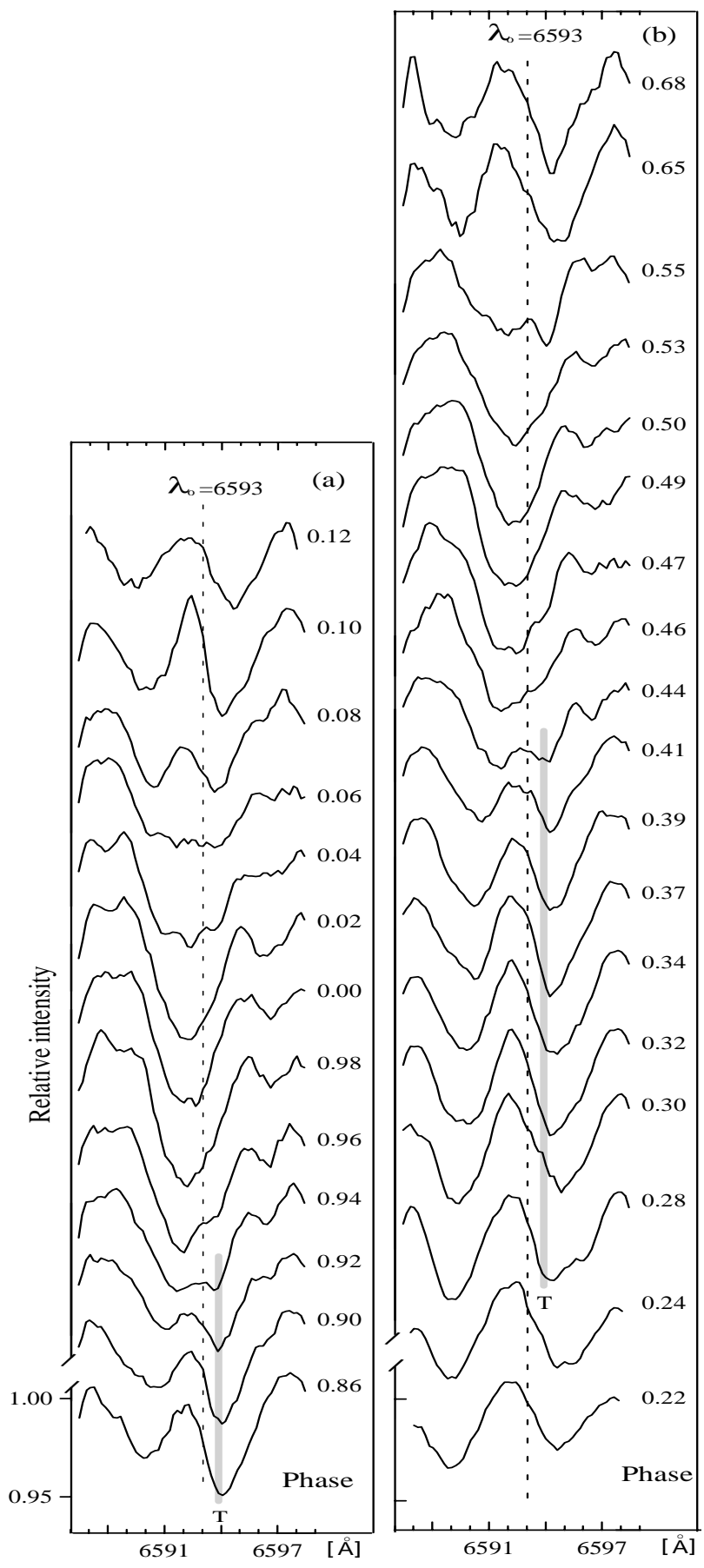

Fig. 8. FeI 6593 profiles of ER Vul from August 14 (a - left panel) and August 15 ( $b$ - right panel). The position of the third feature "T", when visible, is marked with a grey bar.

and originating mainly from the opposite to the cooler companions hemispheres.

Because the emission (or filled-in) cores of the spectral lines originate from the star's chromospheres, we argue that the CaI 6494 line could be considered as an optical indicator of activity of late-type stars, similarly to the CaII H and $\mathrm{K}$ lines, the first Balmer lines and CaII IRT lines (Linsky et al. 1979). Gunn \& Doyle (1997) detected that the CaII IRT lines of ER Vul are filled-in but their excess variations seem to be uncorrelated to the Balmer lines. Our spectral data also show excess emission 
Table 1. Radial velocity data in $\mathrm{km} \mathrm{s}^{-1}$.

\begin{tabular}{|c|c|c|c|c|c|}
\hline $\begin{array}{r}\text { HJD } \\
2452130+ \\
\end{array}$ & phase & $\begin{array}{l}V_{1}\left(\mathrm{H}_{\alpha}\right) \\
\mathrm{km} \mathrm{s}^{-1}\end{array}$ & $\begin{array}{l}V_{2}\left(\mathrm{H}_{\alpha}\right) \\
\mathrm{km} \mathrm{s}^{-1}\end{array}$ & $\begin{array}{l}V_{1}(\mathrm{Fe}) \\
\mathrm{km} \mathrm{s}^{-1}\end{array}$ & $\begin{array}{l}V_{2}(\mathrm{Fe}) \\
\mathrm{km} \mathrm{s}^{-1}\end{array}$ \\
\hline 6.308458 & 0.820 & 49.7 & -192.8 & 65.4 & -211.2 \\
\hline 6.325125 & 0.844 & 46.1 & -181.6 & 49.3 & -199.7 \\
\hline 6.339012 & 0.864 & 39.2 & -165.1 & 31.1 & -188.0 \\
\hline 6.362625 & 0.898 & 34.7 & -147.7 & 8.3 & -174.0 \\
\hline 6.376512 & 0.918 & 24.0 & -133.7 & & \\
\hline 6.433458 & 0.999 & & & -68.0 & \\
\hline 6.448042 & 0.020 & -56.3 & & -74.8 & \\
\hline 6.504292 & 0.101 & -162.4 & 57.4 & & \\
\hline 6.518180 & 0.120 & -181.4 & 69.2 & -174.6 & 47.4 \\
\hline 6.576514 & 0.204 & & 74.2 & & \\
\hline 6.590402 & 0.224 & -188.8 & 84.7 & -197.5 & 63.6 \\
\hline 6.605680 & 0.246 & -192.8 & & -208.9 & 71.3 \\
\hline 7.287632 & 0.223 & -196.9 & 70.2 & -178.0 & 85.0 \\
\hline 7.302215 & 0.244 & -192.8 & 77.3 & -177.0 & 87.8 \\
\hline 7.316798 & 0.265 & -187.8 & & & 87.0 \\
\hline 7.329965 & 0.283 & -185.5 & 76.0 & -172.0 & 87.0 \\
\hline 7.344573 & 0.304 & -174.6 & 75.5 & -168.2 & 86.0 \\
\hline 7.358465 & 0.324 & -162.8 & 76.2 & -162.7 & 78.3 \\
\hline 7.373048 & 0.342 & -158.3 & 70.2 & -158.1 & 74.4 \\
\hline 7.386923 & 0.365 & -149.5 & 64.1 & -149.9 & 69.6 \\
\hline 7.400798 & 0.385 & -140.5 & 60.3 & -131.6 & 64.0 \\
\hline 7.414715 & 0.405 & -130.3 & 53.3 & & \\
\hline 7.546632 & 0.594 & & & 53.5 & \\
\hline 7.560548 & 0.614 & & & 64.9 & \\
\hline 7.575132 & 0.635 & 65.0 & -169.6 & 76.8 & -140.8 \\
\hline 7.589007 & 0.654 & 75.8 & -185.5 & 83.2 & -170.9 \\
\hline 7.603590 & 0.675 & 87.3 & -196.6 & 90.1 & -181.9 \\
\hline
\end{tabular}

in the cores of the CaI 6494 line without clear correlation with the $\mathrm{H}_{\alpha}$ excess emission.

\subsection{Radial velocity solution}

The measurements of the radial velocity of the spectral lines of the short-period, RS CVn-stars are difficult due to their rotational broadening and variable blending with the surrounding metal lines (Frasca et al. 2000). Additionally, the profiles are distorted by emission or absorption features. Nevertheless, Hill et al. (1989) showed that for detached systems with rotational velocity $<100 \mathrm{~km} \mathrm{~s}^{-1}$ (like ER Vul), the Gaussian fit is a good approximation. Moreover, the lines of both stellar components in ER Vul are well separated in our spectra due to their relative high $S / N$ ratios and high resolutions. Therefore, we determined the radial velocities of the $\mathrm{H}_{\alpha}$ and FeI 6593 lines by fitting them with sums of two gaussians at each phase (Fig. 12). The error of the individual measurement do not exceed $25 \mathrm{~km} \mathrm{~s}^{-1}$ for the lines of the primary star and $32 \mathrm{~km} \mathrm{~s}^{-1}$ for the lines of the secondary star. The obtained values of the radial velocities for both lines are given in Table 1. Figure 13 shows our data together with those by Hill et al. (1990) that are phased using the ephemeris (1) and applied correction of $28 \mathrm{~km} \mathrm{~s}^{-1}$ to the systemic velocity. A good agreement between the two samples of data is visible. The difference of $28 \mathrm{~km} \mathrm{~s}^{-1}$ between our $\gamma$ velocity and that of Hill et al. (1990) has an instrumental origin

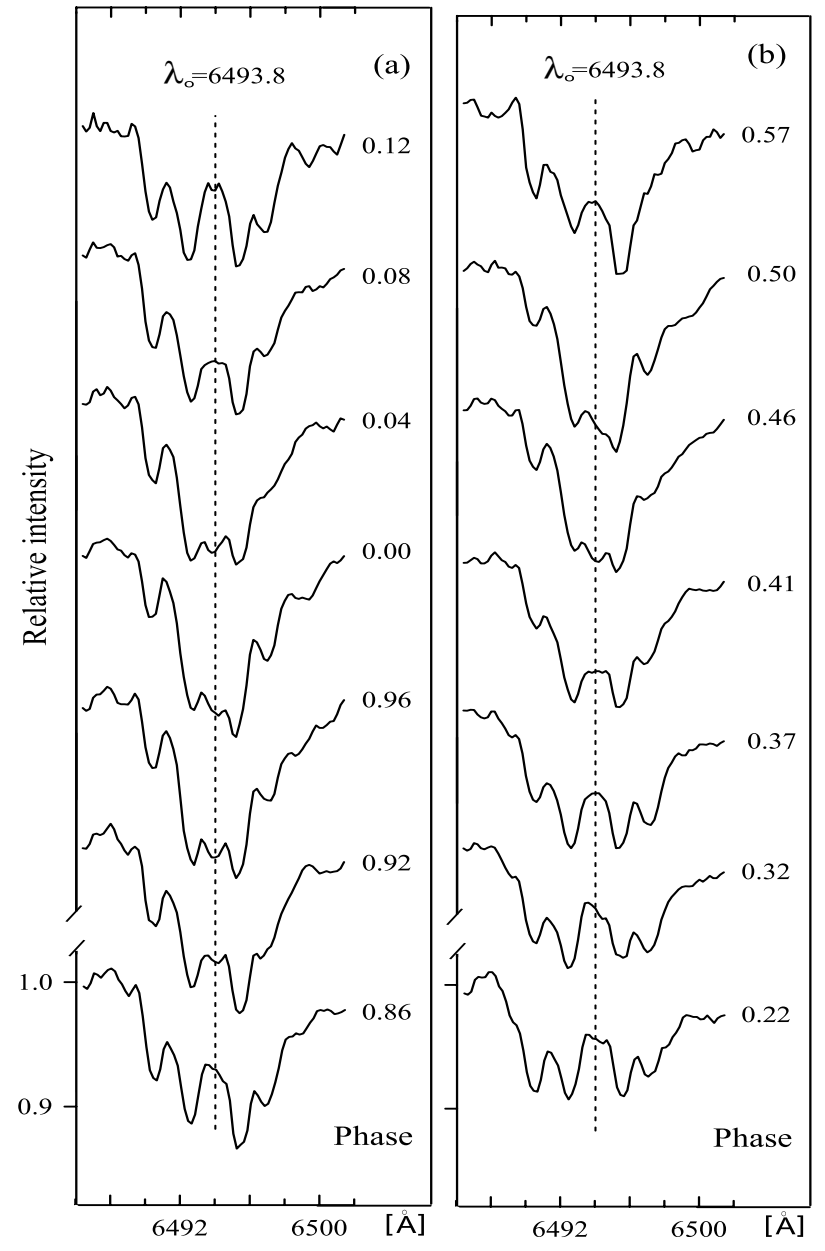

Fig. 9. CaI 6494 profiles of ER Vul from August 14 (a - left panel) and August 15 ( $b$ - right panel).

and it is due to the lack of observations of the radial velocity standards in our runs.

Our radial velocity data were fitted by sinusoids and the results of the least squares fit are: $K_{1}=136 \pm 2.4 \mathrm{~km} \mathrm{~s}^{-1}$ and $K_{2}=149.9 \pm 2.4 \mathrm{~km} \mathrm{~s}^{-1}$. Our $K_{1}$ value is slightly smaller than that of Hill et al. (1990) (139.5 $\left.\mathrm{km} \mathrm{s}^{-1}\right)$ and Northcott \& Bakos (1967) $\left(138.5 \mathrm{~km} \mathrm{~s}^{-1}\right)$ but the same as that of Mclean (1982). Our $K_{2}$ value is slightly bigger than that of Hill et al. (1990) $\left(145.8 \mathrm{~km} \mathrm{~s}^{-1}\right)$ but the same as that of Northcott \& Bakos (1967).

We determined the rotational broadenings of the $\mathrm{H}_{\alpha}$ and FeI 6594 lines for ER Vul with the same method as that used for SV Cam (Kjurkchieva et al. 2002), i.e. fitting the central parts of their profiles by 6th-order polynomial and measuring the half width of these fits at the continuum level. The averaged values for both lines are almost the same: $\Delta \lambda_{1}^{\text {rot }}=1.8 \AA$ and $\Delta \lambda_{2}^{\text {rot }}=1.6 \AA$. The equatorial velocities corresponding to these rotational broadenings and to the determined orbital inclination are $V_{1}^{\mathrm{eq}}=90 \pm 5 \mathrm{~km} \mathrm{~s}^{-1}$ and $V_{2}^{\mathrm{eq}}=80 \pm 5 \mathrm{~km} \mathrm{~s}^{-1}$. Our values of the equatorial velocities are bigger than those determined by Hill et al. (1990): $V_{1}^{\mathrm{eq}}=81 \mathrm{~km} \mathrm{~s}^{-1}$ and $V_{2}^{\mathrm{eq}}=71 \mathrm{~km} \mathrm{~s}^{-1}$. 

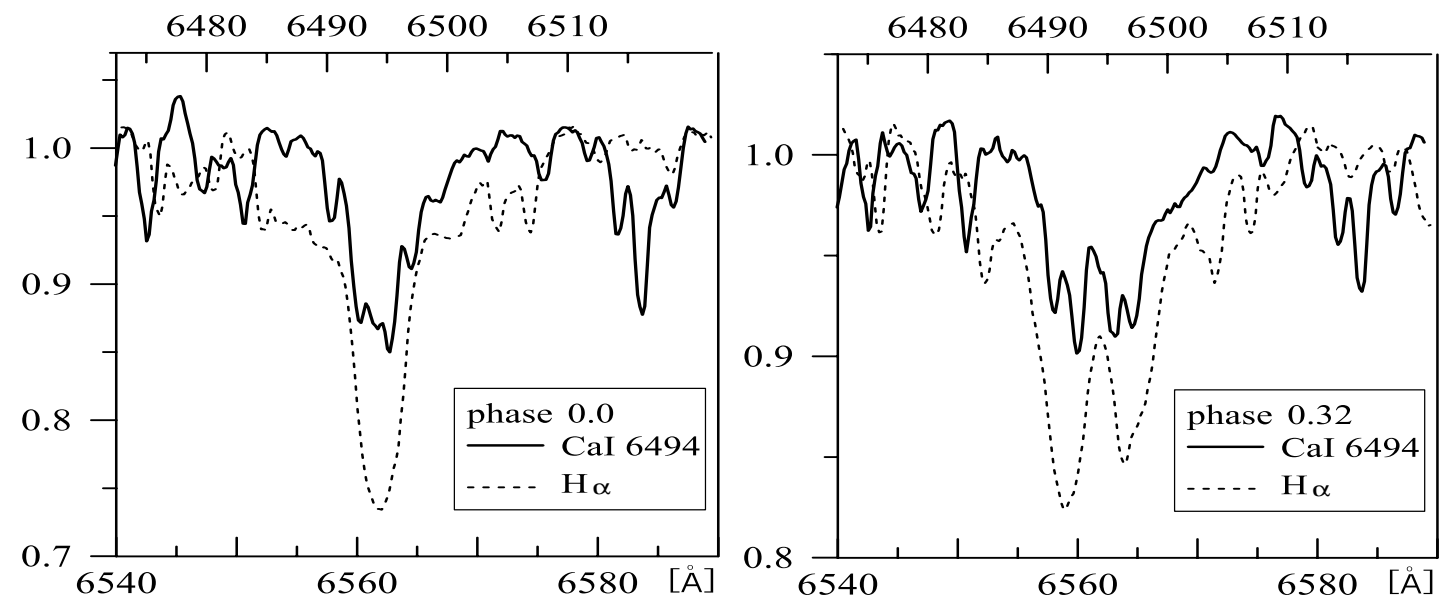

Fig. 10. The similarity of the profiles of the $\mathrm{H}_{\alpha}$ and $\mathrm{CaI} 6494$ lines at phases 0.0 and 0.32 .
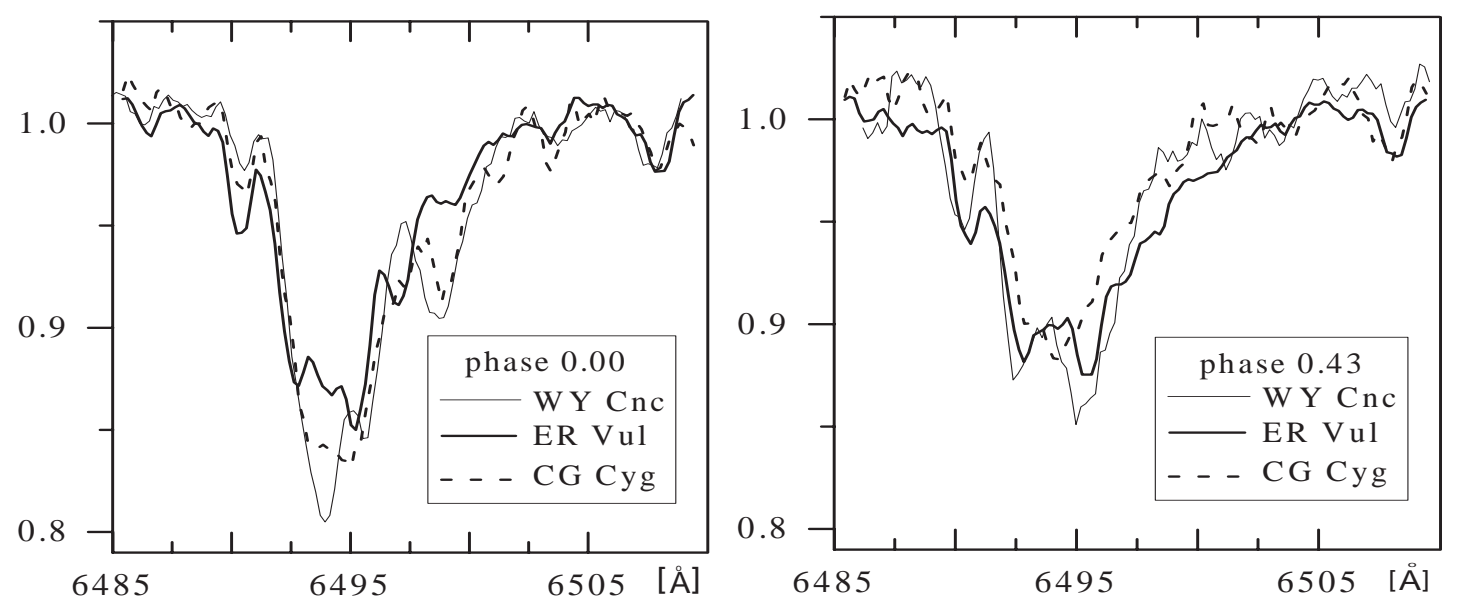

Fig. 11. The CaI 6494 line in the spectra of the short-period RS CVn stars: CG Cyg, ER Vul and WY Cnc, shown in the middle of the primary eclipse (left panel) and before the secondary minimum (right panel).

\subsection{Analysis of the photometric data}

Our new light curve of ER Vul shows the following peculiarities:

(a) The decreasing branch of the secondary minimum is steeper than the increasing one;

(b) The brightness around the second quadrature is smaller than that at the first one. This effect is more apparent in $V$ and $R$ colors than in $B$;

(c) There are standstills before and after the secondary eclipse.

Color indices of ER Vul change very slightly during the orbital cycle. The color index $B-V=0.59$ is precisely the same as that determined by Hill et al. (1990) and corresponds to the spectral types of the components.

Usually the distortions of the light curves of the shortperiod, RS CVn stars are reproduced successfully with cool, photospheric spots. Most researchers place $\operatorname{spot}(\mathrm{s})$ on the primary star which, in many systems, is the dominant luminosity source. In case of ER Vul, however, the contributions to the total system luminosity of both components are close. That is why Hill et al. (1990) and Olah et al. (1994) noted that the light curves of ER Vul can be reproduced equally well by spots

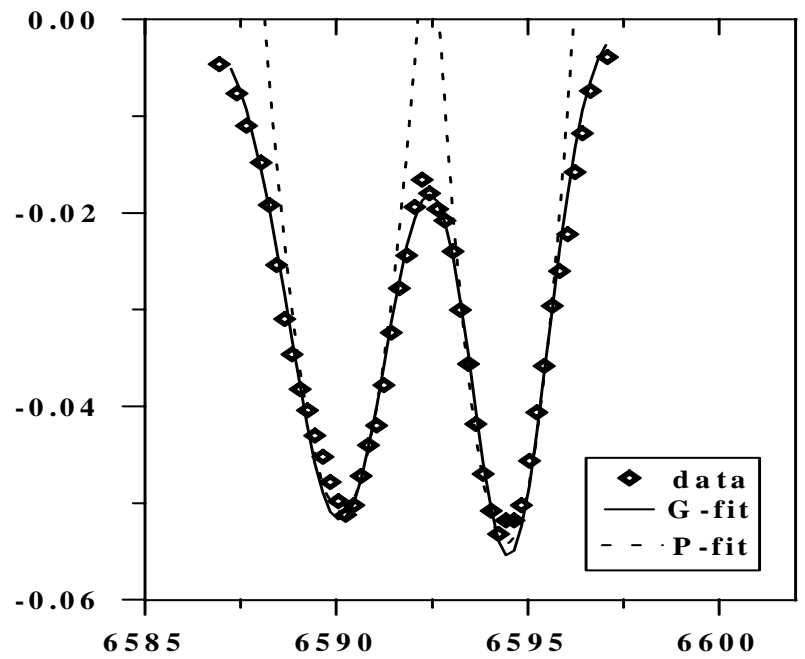

Fig. 12. The FeI $6593 \AA$ A line shown both with a Gaussian fit (G-fit) for the radial velocity measurements and a polynomial fit (P-fit) for the equatorial velocity measurements.

either on the primary or on the secondary component. However, Piskunov (1996) analyzing the Doppler images showed that 


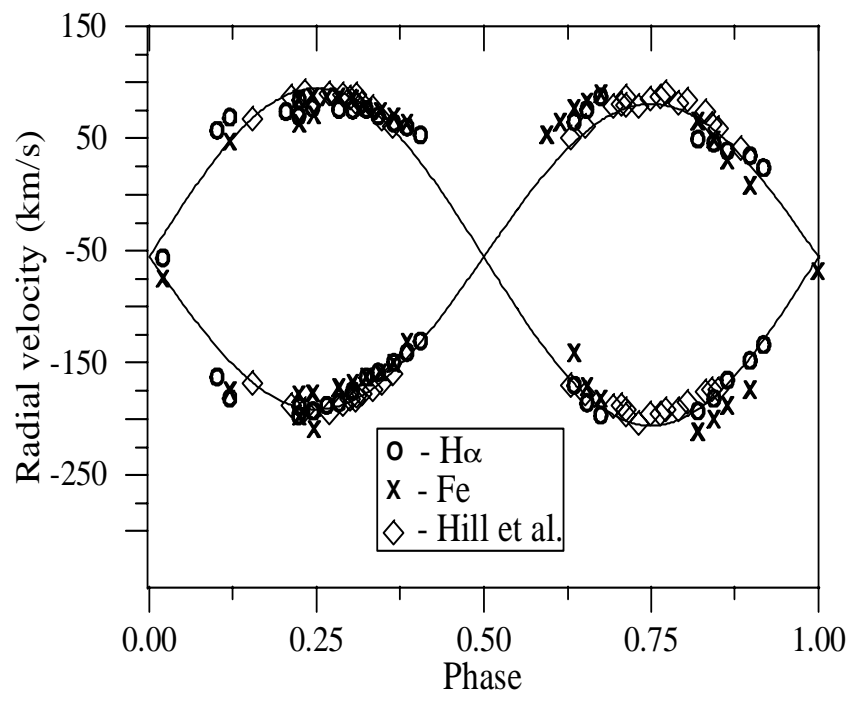

Fig. 13. Radial velocity curves of ER Vul.

both stars in ER Vul are covered with spots. Modelling of light curves distorted by spots may fail to get reliable spots' parameters due to the similarity of the components (Olah et al. 1996). Another difficulty is the small amplitudes of the light variability due to the low orbital inclination.

In order to re-determine the physical parameters of components we performed the light curve modeling with the WilsonDevinney code ver. 1996 (Wilson 1979; Wilson 1993) but we applied the Monte Carlo algorithm as the search method (Zola et al. 1997). In our light curve solution we fixed only the mass ratio $q=0.91$ (determined by our radial velocity solution) and the primary star's temperature $T_{1}=6000 \mathrm{~K}$, corresponding to its spectral type. The theoretical values of both albedo and gravity darkening coefficients were used. The limb darkening coefficients were adopted as functions of the temperature and wavelength from Díaz-Cordovés et al. (1995) and Claret et al. (1995) tables.

Since our light curve is heavily distorted, with the goal to determine reliably the system parameters, we included two cool spots placed on the primary star's surface to remove the observed distortion during the search. A reasonably good fit to the observations was obtained with two spots located on the opposite hemispheres and covering about $6 \%$ of the primary component surface. There is, however, some discrepancy between observations and the synthetic light curves (see Fig. 14). We can propose two reasons for such a poor fit: first, the discrepancy can be due to a simplified spot model in the W-D code and, second, the intrinsic, night-to-night variability of the light curve due to changes of spots properties (sizes, temperature or location). The parameters resulting from our solution are presented in Table 2 along with these derived by other authors. The errors quoted for our solution are the standard errors calculated for free parameters from all elements in the search arrays at the end of search for 6 independent search runs. These runs were done for three sets of normal points and using two weighting: proportional to 1 /flux and proportional to $1 /$ flux $^{2}$.

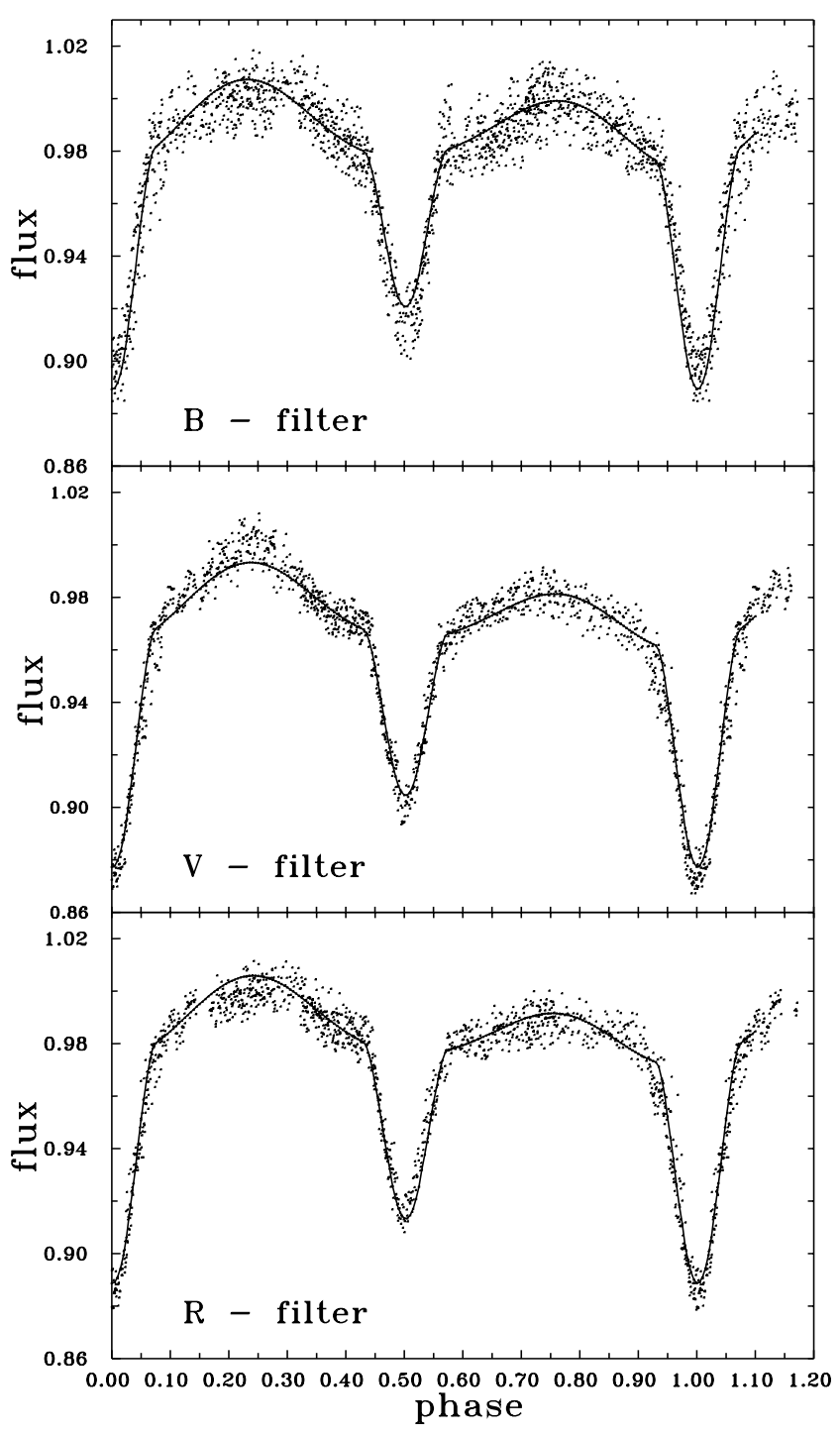

Fig. 14. Theoretical light curves (solid lines) versus observations of ER Vul (dots). The synthetic light curve corresponds to our best solution with two cool spots on the primary component.

\section{Global parameters and signs of activity}

Making use of photometrically determined orbital inclination $\left(i=66.1^{\circ}\right)$ and our values of $K_{1}$ and $K_{2}$, we re-determined the masses of components: $M_{1}=1.16 \pm 0.04 M_{\odot}$ and $M_{2}=$ $1.05 \pm 0.04 M_{\odot}$.

On the basis of our radial velocity solution and the relative star's radii $r_{1}=0.299$ and $r_{2}=0.269$, we obtained the absolute radii: $R_{1}=1.25 \pm 0.05 R_{\odot}$ and $R_{2}=1.12 \pm 0.16 R_{\odot}$. Their ratio $k=R_{2} / R_{1}=0.896$ is equal to the ratio of the stellar equatorial velocities $p=V_{2}^{\mathrm{eq}} / V_{1}^{\mathrm{eq}}=0.89$.

The radii of the MS stars, inferred from the mass-radius relations and corresponding to the above masses, would be: $R_{1}=1.14 R_{\odot}$ and $R_{2}=1.04 R_{\odot}$, indicating that both components of ER Vul are oversized for their masses. The relative radii of the Roche volumes corresponding to the mass ratio obtained in this paper are: $R_{\mathrm{L} 1}=0.36$ and $R_{\mathrm{L} 2}=0.347$, therefore, both stars fill about half of their Roche lobes. 
Table 2. Global parameters of ER Vul.

\begin{tabular}{cccccccc}
\hline \hline$i$ & $q$ & $r_{1}$ & $r_{2}$ & $T_{1}$ & $T_{2}$ & $l_{1}(V)$ & Ref. \\
\hline $68.5^{\circ} \pm 0.6$ & & $0.259 \pm 0.009$ & $0.252 \pm 0.019$ & $6100 \mathrm{~K}$ & $5800 \mathrm{~K}$ & $0.559 \pm 0.031$ & $1^{*}$ \\
$67^{\circ}$ & & 0.281 & 0.274 & $6050 \mathrm{~K}$ & $6000 \mathrm{~K}$ & 0.57 & $2^{* *}$ \\
$69^{\circ}$ & & 0.299 & 0.281 & $6000 \mathrm{~K}$ & $5520 \mathrm{~K}$ & 0.54 & $3^{* *}$ \\
$71.6^{\circ}$ & 0.93 & 0.254 & 0.204 & $6100 \mathrm{~K}$ & $5800 \mathrm{~K}$ & 0.65 & 4 \\
$69.0^{\circ} \pm 0.3$ & & $0.258 \pm 0.006$ & $0.239 \pm 0.01$ & $6100 \mathrm{~K}$ & $5800 \mathrm{~K}$ & $0.561 \pm 0.013$ & 5 \\
$69.1^{\circ} \pm 0.6$ & $0.886 \pm 0.025$ & 0.278 & 0.220 & $6000 \pm 58 \mathrm{~K}$ & $5696 \pm 70 \mathrm{~K}$ & 0.668 & 6 \\
$66.7^{\circ} \pm 0.3$ & 0.95 & $0.25 \pm 0.01$ & $0.25 \pm 0.01$ & $5900 \pm 100 \mathrm{~K}$ & $5750 \pm 200 \mathrm{~K}$ & 0.647 & 7 \\
$66.7^{\circ} \pm 0.22$ & 0.96 & $0.2858 \pm 0.0051$ & $0.2626 \pm 0.0152$ & $6000 \mathrm{~K}$ & $5808 \pm 36 \mathrm{~K}$ & 0.53 & 8 \\
$67.7^{\circ} \pm 0.4$ & 0.957 & $0.260 \pm 0.008$ & $0.259 \pm 0.023$ & 6100 & 5800 & 0.56 & 9 \\
$66.1^{\circ} \pm 1.1$ & $0.906 \pm 0.030$ & $0.299 \pm 0.003$ & $0.269 \pm 0.015$ & $6000 \mathrm{~K}$ & $5514 \pm 32 \mathrm{~K}$ & $0.654 \pm 0.043$ & 10 \\
\hline
\end{tabular}

References: 1 - Abrami \& Cester (1963); 2 - Hrivnak (1980); 3 - Al-Naimly (1981); 4 - Budding \& Zelik (1987); 5 - Olah \& Budding (1993); 6 - Mennella (1990); 7 - Hill et al. (1990); 8 - Ibanoglu et al.(1993); 9 - Olah et al. (1994); 10 - this paper.

Note: * - light curve solution by Olah \& Budding (1993); ** - light curve solution by Hill et al. (1990).

Table 2 presents the global parameters of ER Vul determined by different authors. It shows a good agreement of our results with those from previous studies.

Our results confirm some appearances of activity inferred by previous investigations in the optical range and provide new spectral indications of this activity.

We divided the signs of activity observed in ER Vul into two types: first, due to the presence of circumstellar matter being a result of some active processes, and, second, photospheric and chromospheric activity of the stars themselves.

As an appearance of the first type of activity, we consider the presence of the absorption feature between the two stellar profiles that is visible in the $\mathrm{H}_{\alpha}$ and FeI 6593 lines out of the eclipses. It may be attributed to cool matter around the center of mass of the binary system or near the Lagrangian point $L_{1}$ (for mass ratio $q=0.9$ they are very close). Such circumstellar gas could be a result of different mechanisms of mass loss from the stellar components. Northcott \& Bakos (1967), McLean (1982) and Hill et al. (1990) also suggested the presence of a gaseous cloud at the inner Lagrangian point in order to explain the phase behavior of the spectral lines of ER Vul. Arevalo et al. (1988) suggested the existence of such a structure to explain the observed IR and UV excess of ER Vul.

The apearances of the second type of activity could be seen in the following observational data:

(a) The emission feature E (Fig. 5) on the blue wing of the $\mathrm{H}_{\alpha}$ line appearing around the eclipse minima may be due to structures extended above the stellar limbs (prominence-like material co-rotating with one of the components). On the other hand, the observed systematic blue shifts in the $\mathrm{H}_{\alpha}$ emission of the Sun are interpreted as a result of spicules;

(b) The profiles of the $\mathrm{H}_{\alpha}$ line of both stellar components are filled-in by an emission of chromospheric origin;

(c) The profiles of the CaI 6494 line (broad absorption with central emission feature) seem similar to those of collisional dominated resonance CaII $\mathrm{H}$ and $\mathrm{K}$ lines seen in active stars and we suppose that the central emission of the CaI 6494 lines can be a sign of the chromospheric activity of ER Vul.

\section{Conclusions}

The main results of our spectroscopic and photometric observations and their analysis can be summarized as follows:

(1) By simultaneous solution of the radial velocity curves and multicolor light curve we re-determined the global parameters of the binary system ER Vul (masses, radii, temperatures, equatorial velocities and orbital inclination). The obtained values are in a good agreement with those determined by other authors.

(2) Our spectral data evidenced different appearances of the activity of the star which confirm previous results as well as predictions of the magnetic-dynamo theory.

$\mathrm{X}$-ray and UV studies of RS CVn stars suggest that there is a level at which the emission becomes saturated (Vilhu \& Rucinski 1983). The suspicion that ER Vul is close to the saturation limit for chromospheric activity is based on the very large filling factor obtained by Gunn \& Doyle (1997), the very high level of X-ray, UV and radio emission as well as the excess emission in the $\mathrm{H}_{\alpha}$, CaII IRT and MgI b lines (indicative for non-radiative energy dissipation in thick chromospheres). Our observations provide new spectral indications of the high level of activity of ER Vul.

Acknowledgements. This research was supported in part by the NATO Linkage grant No. PST.CLG.978810 and grant No. 1/2002 of the Shoumen University. The Monte Carlo computations were performed at ACK "Cyfronet" in Cracow under grant No. KBN/UJ/015/95, which we gratefully acknowledge. The authors are grateful to an anonymous referee for the useful advices and suggestions that let to significant improvement of the paper.

\section{References}

Abrami, A., \& Cester, B. 1963, Contr. Oss. Astron. Trieste No. 320 Al-Naimly, H. 1981, A\&AS, 43, 85

Arevalo, M., Lazaro, C., \& Fuensalida, J. 1988, AJ, 96, 1061

Borkowski, J. 1988, Internal Report of the Astronomical Institute in Torun (in Polish)

Budding, E., \& Zeilik, M. 1987, ApJ, 319, 827 
Claret, A., Díaz-Cordovés, J., \& Gimenez, A. 1995, A\&AS, 114, 247

Díaz-Cordovés, J., Claret, A., \& Gimenez, A. 1995, A\&AS, 110, 329

Frasca, A., \& Catalano, S. 1994, A\&A, 284, 883

Frasca, A., Marino, G., Catalano, S., \& Marilli, E. 2000, A\&A, 358, 1007

Gray, D. 1992, in The observations and analysis of stellar photospheres, Cambridge Astrophys. Ser., 20, 248

Gunn, A., \& Doyle, J. 1997, A\&A, 318, 60

Hall, D. 1976, in Multiple Periodic Variable Stars, ed. W. Fitch, (Dordrecht: Reidel), 287

Hill, G., Fisher, W., \& Holmgren, D. 1989, A\&A, 218, 152

Hill, G., Fisher, W., \& Holmgren, D. 1990, A\&A, 238, 145

Hrivnak, B. 1980, Ph.D. Thesis, University of Pennsylvania

Ibanoglu, C., Evren, S., Akan, M., Tunca, Z., \& Keskin, V. 1993, A\&A, 269, 310

Kjurkchieva, D., Marchev, D., \& Ogloza, W. 2001, A\&A, 378, 102

Kjurkchieva, D., Marchev, D., \& Zola, S. 2002, A\&A, 386, 548

Kjurkchieva, D., Marchev, D., \& Ogloza, W. 2003, A\&A, in press

Kreiner, J., Krzesinski, J., Pokrzywka, B., et al. 1993, IAU Coll. 136, Poster Papers on Stellar Photometry, ed. I. Elliot, \& C. Butler (Dublin Institute for Advanced Studies), 80

Kreiner, J., Kim, C., \& Nha Il-Seong 2001, An Atlas of O-C Diagrams of Eclipsing Binary Stars (Krakow Pedagogical University Press)

Krzesinski, J., \& Wojcik, K. 1993, A\&A, 280, 338

Lazaro, C., \& Arevalo, M. 1997, AJ, 113, 2283

Linsky, J., Hunten, D., Sowell, R., Glackin, D., \& Kelch, W. 1979, ApJS, 41, 481
McLean, B. 1982, MNRAS, 201, 421

Mennella, V. 1990, A\&A, 234, 203

Montes, D., Fernandez-Figueroa, M., De Castro, E., \& Cornide, M. 1995, A\&A, 294, 165

Northcott, R., \& Bakos, G. 1967, AJ, 72, 89

Olah, K., \& Budding, E. 1993, New Frontiers in Binary Star Research, ASP Conf. Ser., 38, ed. K.-C. Leung, \& I.-S. Nha

Olah, K., Budding, E., Kim, H., \& Etzel, P. 1994, A\&A, 291, 110

Olah, K., Kovari, Z., \& Guinan, E. 1996, Poster Proccedings of IAU Symp. 176, ed. K. Strassmeier, 159

Piskunov, N. 1996, IAU Symp. 176, Proceedings, ed. K. Strassmeier, \& J. Linsky, 45

Rucinski, S. 1998, AJ, 115, 303

Smirnov, O., Piskunov, N., Afanasyev, V., \& Morozov, A. 1992, ASP Conf. Ser., 26, Astron. Data Analysis software and Systems, ed. D. Worrall et al., 344

Strassmeier, K., Fekel, F., Bopp, B., Dempsey, R., \& Henri, G. 1990, ApJS, 72, 191

Vilhu, O., \& Rucinski, S. M. 1983, A\&A, 127, 5

Wilson, R. 1993, Documentation of the Eclipsing Binary Computer Model

Zarro, D., \& Rogers, A. 1983, ApJS, 53, 815

Zeinali, F., Edalati, M. T., \& Mirtorabi, M. T. 1995, IBVS No. 4190

Zola, S., Kolonko, M., \& Szczech, M. 1997, A\&A, 324, 1010 